\title{
Guia de Técnicas para a Gestão do Tempo de Estudos: Relato da Construção
}

\author{
Fabiane Cristina Pereira Marcilio ${ }^{1}$ \\ ${ }^{1}$ Universidade Federal do Rio Grande do Sul, RS, Brasil. \\ Alessandra Blando ${ }^{1}$ \\ ${ }^{1}$ Universidade Federal do Rio Grande do Sul, RS, Brasil.
}

\author{
Roberta Zanini da Rocha ${ }^{1}$ \\ ${ }^{1}$ Universidade Federal do Rio Grande do Sul, RS, Brasil. \\ Ana Cristina Garcia Dias ${ }^{1}$ \\ ${ }^{1}$ Universidade Federal do Rio Grande do Sul, RS, Brasil.
}

Resumo: Uma das dificuldades com as quais os universitários deparam ao iniciar o curso superior é a forma de administrar o tempo, interferindo nos estudos e na vida de modo geral. Em função disso, considera-se importante que o profissional que irá auxiliá-lo em sua gestão de tempo consiga compreender como ocorre o processo de orientação em relação a esse tema. O objetivo deste trabalho consiste em descrever a criação e a utilização do Guia de técnicas para gestão do tempo de estudos na universidade, que visa auxiliar os profissionais na prática de atendimento a universitários que apresentem dificuldades na administração do tempo de estudos. Esse material, desenvolvido nos anos de 2017 e 2018, proporciona sugestões de dezesseis técnicas apresentando em cada uma seu objetivo, os materiais necessários e a descrição da aplicação. As técnicas estão subdivididas de acordo com quatro etapas: a) diagnóstico do uso do tempo; b) desenvolvimento de estratégias para lidar com as dificuldades; c) estabelecimento de objetivos e metas; e d) implementação e avaliação das mudanças. Observou-se que as intervenções, apoiadas pela utilização do guia têm auxiliado os alunos com dificuldades na gestão do tempo que frequentam um serviço de apoio aos estudantes universitários.

Palavras-chave: Guia de Técnicas, Gestão do tempo de Estudos, Aprendizagem, Universitários.

\section{Guide of Techniques for Study Time Management: Report of Construction}

\begin{abstract}
One of the difficulties that college students face when they start college is how to manage time, what interferes in the studies and life in general. Due to this situation, it is important that the professional who will assist the student in his time management can understand how the orientation process occurs in relation to this topic. The aim of this work is to describe the creation and use of the Guide to Techniques for Time Management of Studies in the University. It intends to assist professionals in the practice of attending university students who have difficulties in the management of study time. This material, developed in the years 2017 and 2018, presents an intervention composed of sixteen techniques, (the purposes of each technique, necessary materials and the application guidelines). The techniques should be applied in four stages: a) diagnosis of the use of time; b) development of coping strategies; c) setting goals and targets; and d) implementation and evaluation of changes. It was noted that the interventions, supported by the use of the guide, have helped the students that attend a service of support to university students in their difficulties with the management of their time.
\end{abstract}

Keywords: Guide to Techniques, Time Management of Studies, Learning, University Students. 


\title{
Guía de Técnicas para la Gestión del Tiempo de Estudios: Relato de la Construcción
}

\begin{abstract}
Resumen: Una de las dificultades que los universitarios enfrentan al iniciar un curso superior es la manera de administrar el tiempo, interfiriendo en los estudios y en la vida en general. Por eso, se consideraimportantequeelprofesional queles asista conlagestión deltiempo consiga comprender cómo ocurre el proceso de orientación en relación a ese tema. El objetivo de este trabajo consiste en describir la creación y la utilización de la Guia de Técnicas para Gestão do Tempo de Estudos na Universidade (Guía de Técnicas para la Gestión del Tiempo de Estudio en la Universidad) que tiene como fin auxiliar a los profesionales en la práctica de atención a universitarios que presenten dificultades en la administración del tiempo. Este material, desarrollado en 2017 y 2018, presenta una intervención con dieciséis técnicas (objetivo de cada una, materiales necesarios y orientaciones para la aplicación). Se propone que las mismas se apliquen en cuatro etapas: a) diagnóstico del uso del tiempo; b) desarrollo de estrategias para enfrentar las dificultades; $c$ ) establecimiento de objetivos y metas; y d) implementación y evaluación de los cambios. Se observó que las intervenciones, apoyadas por la utilización de la guía, han ayudado a los alumnos que asisten a un servicio de apoyo a estudiantes universitarios en sus dificultades en la gestión del tiempo.
\end{abstract}

Palabras clave: Guía de Técnicas, Gestión del Tiempo de Estudios, Aprendizaje, Universitarios.

Ao ingressar no ensino superior, o estudante depara com uma série de mudanças e desafios para lidar com as exigências desse novo contexto. Esse processo influencia o desenvolvimento do indivíduo, já que ocorre, geralmente, em um momento em que está ficando mais independente não só em relação aos seus estudos, mas também em outras esferas da vida, por estar se tornando adulto (Teixeira, Dias, Wottrich, \& Oliveira, 2008). Assim, a implementação de mudanças e adaptações (de crenças, hábitos e/ou comportamentos) é fundamental para que o acadêmico consiga se integrar adequadamente à universidade e às tarefas da vida adulta (Ding, 2017).

Um dos aspectos envolvidos nessa transição é o desenvolvimento da autonomia, requerida principalmente diante de um ambiente menos estruturado como o universitário. No ensino médio, o adolescente passava a maior parte do tempo em função da escola, ambiente no qual, em geral, se realiza um maior monitoramento do desempenho e dos comportamentos do estudante, sob pena de sanções e comunicados aos pais. A universidade, por outro lado, demanda que o processo de aprendizagem e as condutas passem a ser geridas pelo próprio aluno (Briggs, Clark, \& Hall, 2012; Pinho, Bastos, Dourado, \& Ribeiro, 2013; Teixeira et al., 2008). Espera-se que os estudantes universitários assumam novas responsabilidades, sendo mais ativos e independentes no gerenciamento e organização dos seus estudos e afazeres (Ding, 2017).

O contexto universitário também se diferencia do ambiente escolar por demandar do aluno maior dedicação aos estudos, visto que o nível de exigência e a complexidade dos processos de aprendizagem são maiores. A gestão do tempo, nesse contexto, torna-se uma das competências essenciais a serem desenvolvidas pelos indivíduos, tanto em função das tarefas acadêmicas como de outras áreas da vida (García-Ros \& PérezGonzáles, 2012; Thibodeaux, Deutsch, Kitsantas, \& Winsler, 2017). Uma das diferenças verificadas entre os alunos que apresentam baixo rendimento acadêmico e aqueles que obtêm bom desempenho é justamente a capacidade de organização do tempo de estudos, que lhe permite enfrentar os desafios presentes nesse novo contexto e fase de vida (Umerenkova \& Flores, 2018; Veiga \& Melim, 2007). O desempenho acadêmico, representado pelas notas obtidas nas avaliações, é considerado uma medida da aprendizagem e, ao mesmo tempo, um indicador da qualidade das instituições de ensino superior. Alunos com melhor desempenho também se caracterizam por conseguirem separar as tarefas de estudo de outras atividades, colocando-as em espaços específicos em suas rotinas semanais (Umerenkova \& Flores, 2018). Um mau gerenciamento do tempo, por sua vez, pode significar não conciliar 
aspectos da vida pessoal com os acadêmicos. Não é incomum que o estudante adote diferentes comportamentos disfuncionais nesse período, como estratégias de enfrentamento inadequadas ou comportamentos de procrastinação (Blando, 2015; Oliveira, Carlotto, Teixeira, \& Dias, 2016).

A gestão do tempo é entendida como um processo dirigido a determinados propósitos, que envolvem a avaliação do uso do tempo, o estabelecimento de metas, planejamento, acompanhamento e priorização de tarefas a fim de alcançar objetivos propostos (Claessens, Van Eerde, Rutte, \& Roe, 2007; García-Ros, PérezGonzález, Talaya, \& Martínez, 2008). Cabe lembrar que o tempo é um recurso limitado e disponível da mesma forma para todas as pessoas. Nesse sentido, pode-se dizer que não é o tempo em si que o indivíduo administra, mas sim suas ações e o modo como ele as organiza diante das diferentes tarefas pelas quais é responsável em determinado período de tempo (Claessens et al. 2007).

$\mathrm{Na}$ vida, o comportamento de administrar efetivamente o tempo implica diferentes componentes, que incluem: consciência do tempo necessário para realizar as tarefas rotineiras, identificação dos fatores que podem influenciar o rendimento nas atividades (humor, energia, atenção etc.), utilização de fontes externas para organizar o tempo (caderno de atividades, listas, agenda etc.), desenvolvimento de rotinas e hábitos que incluam tempo para recuperação e descanso, planejamento e determinação de sequências para as tarefas e automonitoramento dos resultados alcançados (White, Riley, \& Flom, 2013).

Especificamente no contexto universitário, manejar adequadamente a realização de atividades implica a capacidade do estudante de avaliar o modo como está usando o próprio tempo de forma a adaptar-se às diferentes demandas acadêmicas. É um processo que envolve o estabelecimento de metas claras, considerando o tempo disponível para tarefas acadêmicas e demais tarefas presentes nesse período (DuránAponte \& Pujol, 2013; Umerenkova \& Flores, 2018). Estudantes com melhores notas acadêmicas tendem a estabelecer metas, estimar o tempo necessário para o desenvolvimento das tarefas, além de possuírem uma rotina de estudos organizada. Esses jovens também costumam avaliar o progresso obtido no processo de aprendizagem (Thibodeaux et al., 2017).

Aliteraturaindicaque, para quehaja gerenciamento efetivo do tempo dedicado aos estudos, são necessários: a) reflexão e definição de objetivos acadêmicos que se deseja alcançar; b) avaliação do tempo disponível para poder organizar as próprias competências, responsabilidades e atividades que se ajustam aos dados de sua realidade (tempo e recursos); c) capacidade de planejamento no estabelecimento de metas específicas de estudo, definindo como irá alcançá-las, priorizando as tarefas a serem realizadas; e, por fim, d) monitoramento e análise dos resultados alcançados, observando o uso do tempo nas diferentes atividades e identificando os fatores e as barreiras que estão atrapalhando o alcance dos objetivos, a fim de propor mudanças nas estratégias ou na organização individual, quando necessárias (García-Ros \& Pérez-Gonzáles, 2012).

Assim, entende-se que administrar o tempo durante a graduação não se restringe simplesmente ao preenchimento de uma planilha com horários de estudos a serem cumpridos ou mesmo a uma prática mecânica que pode ser treinada. $O$ gerenciamento do tempo acadêmico é um processo complexo e amplo que requer do estudante reflexão e avaliação das ações realizadas no presente e metas estabelecidas para o futuro (Blando, 2015). Desse modo, é importante que o aluno reflita constantemente sobre seu processo de aprendizagem, suas demandas (pessoais e acadêmicas) e características presentes no contexto. O indivíduo precisa avaliar o tempo que tem disponível, quais metas precisam ser cumpridas e como ele pode avaliar ou não se conseguiu o sucesso esperado no desenvolvimento delas (Zoltowski, 2016).

Diante dos desafios para lidar com as demandas acadêmicas e as demais tarefas de vida enfrentadas pelo estudante universitário, a oferta de ações voltadas ao desenvolvimento psicossocial e psicopedagógico é essencial (Pavão \& Cezar, 2015). Verifica-se que atividades de cunho psicopedagógico que visam auxiliar os estudantes em suas dificuldades acadêmicas, tais como organização, planejamento e desenvolvimento de estratégias de estudo, assim como de gestão do tempo, geram resultados positivos na qualidade da aprendizagem e na vida pessoal (Basso, Graf, Lima, Schmidt, \& Bardagi, 2013).

O serviço-escola Núcleo de Apoio ao Estudante (NAE), da Universidade Federal do Rio Grande do Sul (UFRGS), oferece ações individuais e grupais destinadas ao manejo do tempo de universitários, especialmente nas ações que envolvem orientação à aprendizagem, terminologia criada no próprio serviço. Esse tipo de orientação, de cunho psicopedagógico, busca desenvolver e aperfeiçoar habilidades e competências 
(incluindo a gestão do tempo), com o objetivo de proporcionar ao aluno maior eficácia em sua aprendizagem e em seu desempenho acadêmico (Blando, 2015).

Nesses atendimentos de orientação à aprendizagem, tanto individuais quanto grupais, observaram-se queixas frequentes relacionadas à administração do tempo de estudos. Isso levou ao desenvolvimento de um método de intervenção e guia com sugestões de técnicas destinadas a profissionais que atendem estudantes com demandas na gestão de tempo. Este trabalho tem por objetivo descrever a criação e utilização desse material, intitulado Guia de técnicas para gestão do tempo de estudos na universidade.

\section{Método}

O Guia de técnicas para gestão do tempo de estudos na universidade foi desenvolvido a partir de experiências práticas de atendimentos individuais e grupais em orientação à aprendizagem no NAE. Para sua construção, primeiramente, realizou-se um levantamento das técnicas de gestão de tempo utilizadas no serviço e na literatura sobre o fenômeno. No serviço, existiam apenas três instrumentos que trabalhavam com esse tema. Percebeu-se, então, a necessidade de criar novas técnicas que fossem específicas sobre o gerenciamento do tempo de estudos, além de um material descritivo sobre a aplicação dessas técnicas com a proposta de auxiliar os profissionais da equipe nos atendimentos. Ao longo das descrições das técnicas, foi possível agrupá-las em diferentes etapas, criando-se um método de como atender universitários com dificuldades de organização de seus estudos. Algumas das técnicas foram adaptadas do livro A arte de planejar o tempo (Wagner, 2003), que apresenta dicas de como realizar um planejamento pessoal, enfocando diferentes dimensões da vida. O processo de criação do guia demandou da equipe do serviço diversas reuniões para analisar e discutir o material. As intervenções em orientação à aprendizagem que seguem as etapas propostas no guia costumam variar, em geral, de quatro a oito encontros de aproximadamente 50 minutos cada.

\section{Resultados}

A construção do guia resultou no desenvolvimento de 16 técnicas, subdivididas em quatro etapas principais de atendimento. O material não tem caráter prescritivo, oferece sugestões de técnicas com a descrição dos objetivos de cada uma, dos materiais necessários para sua aplicação e algumas indicações sobre seu uso. Sua utilização foi planejada para o atendimento individual, entretanto entende-se que podem ser adaptadas para a modalidade grupal. Porém ressalta-se que as etapas devem ser seguidas conforme a ordem apresentada, pois seguem um fluxo lógico para que o indivíduo possa organizar seu tempo de maneira mais eficiente.

Parte-se do pressuposto que, antes de iniciar um planejamento do tempo para os estudos, é necessário compreender o uso do tempo, em geral, realizando um diagnóstico sobre seu uso. A partir dessa análise, é possível criar estratégias para lidar com as possíveis dificuldades identificadas e fortalecer comportamentos que favorecem essa gestão de tempo efetiva. Após esse diagnóstico, pode-se planejar o tempo, considerando objetivos e metas a serem alcançadas. Por fim, trabalha-se com o estudante a implementação do planejamento realizado, para ver quais as dificuldades são encontradas e como estas podem ser remanejadas. O Quadro 1 apresenta as técnicas agrupadas conforme as quatro etapas presentes no guia.

\section{Quadro 1}

Etapas e técnicas do Guia de gestão do tempo de estudos na universidade.

\begin{tabular}{ll}
\hline \multicolumn{1}{c}{ Etapas } & \multicolumn{1}{c}{ Técnicas } \\
\hline 1) Diagnosticar o uso do tempo & 1. Questionário sobre administração do tempo \\
& de estudo \\
& 2. Agenda percebida da semana \\
& 3. Agenda real da semana \\
& 4. Avaliação e registro do \\
& uso do tempo \\
\hline 2) Desenvolver estratégias para lidar & 5. Estratégias para organização do tempo dos estudos \\
com as dificuldades & 6. Checklist para organização \\
& dos estudos \\
& 7. Definindo prioridades: \\
& Urgente x Importante \\
\hline
\end{tabular}


...continuação

\begin{tabular}{ll}
\hline \multicolumn{1}{c}{ Etapas } & \multicolumn{1}{c}{ Técnicas } \\
\hline 3) Estabelecer objetivos e metas & 8. Definindo objetivos \\
& 9. Estabelecendo objetivos \\
& SMART de estudos \\
& 10. Diário de gestão do tempo de estudo \\
& 11. Planejamento de atividades de estudo durante a semana \\
& 12. Modelo de planejamento semanal: lista ABC \\
& 13. Roteiro de estudo para provas \\
\hline 4) Implementar e avaliar mudanças & 14. Roteiro de avaliação da gestão do tempo de estudo \\
& 15. Avaliação do uso do tempo na semana \\
& 16. Avaliação dos objetivos SMART \\
\hline
\end{tabular}

\section{Etapa 1: Diagnóstico do uso do tempo}

Na primeira etapa, o principal objetivo é identificar como o estudante está administrando seu tempo. Deve-se investigar quais são suas principais dificuldades e a avaliação que faz delas. A partir do uso das técnicas sugeridas, $\mathrm{o}$ aluno consegue perceber o tempo dedicado a cada uma de suas atividades realizadas ao longo da semana, incluindo os estudos, tempo de lazer, tempo para deslocamento etc. Muitas dessas atividades não são consideradas quando se faz um planejamento leigo da gestão do tempo. Contudo é muito importante que o indivíduo contabilize essas atividades em seu planejamento geral. Podem ser utilizadas quatro técnicas nesta etapa, abordadas a seguir.

O Questionário de administração do tempo de estudos tem por objetivo auxiliar na identificação dos problemas relacionados à gestão do tempo de modo geral. São apresentadas algumas frases para que o estudante possa verificar a frequência de determinados comportamentos envolvidos na realização das atividades de estudos, especialmente relacionados à procrastinação (exemplos: "deixo os estudos para a véspera da prova" etc.). Considera-se importante identificar e explorar as dificuldades e suas possíveis causas para depois refletir sobre as estratégias a fim de saná-las, mesmo que isso seja feito em um momento posterior.

A Agenda percebida da semana é utilizada para identificar como o estudante percebe sua organização semanal do tempo, buscando investigar qual a compreensão deste em relação ao uso de seu tempo. Nesse momento, o orientador deve advertir para ausência de algumas atividades que consomem o tempo, mas que podem não estar descritas (exemplo: tempo de deslocamento). Por sua vez, a Agenda real da semana propõe-se a verificação de como realmente o tempo está sendo administrado. Solicita-se que o estudante, ao longo da semana, preencha novamente sua agenda semanal com as atividades efetivamente realizadas, incluindo-se, por exemplo, tempo de deslocamento, interrupções, tempo de lazer, de estudo, de uso de redes sociais etc. A Agenda real possibilita constatar o uso real do tempo, comparando com a percepção que o aluno tinha em relação à agenda anterior (a percebida).

A técnica Avaliação e registro do uso do tempo apresenta as orientações para que o estudante faça a Agenda percebida e Agenda real da semana. Após o preenchimento das duas agendas, é solicitado que o aluno escreva as principais diferenças identificadas entre ambas e que realize uma avaliação a partir do levantamento dos pontos considerados positivos e negativos em seu gerenciamento do tempo.

\section{Etapa 2: Desenvolvimento de estratégias para lidar com as dificuldades}

A segunda etapa tem como finalidade auxiliar o universitário a criar estratégias para lidar com as dificuldades percebidas em sua organização do tempo de estudo, especialmente buscando formas de resolvê-las. Para tanto, são utilizadas técnicas que apresentam diferentes estratégias capazes de levar à otimização do processo de aprendizagem. Os indivíduos devem avaliar, entre as estratégias apresentadas, aquelas que se adaptam melhor a sua realidade e que podem contribuir para a eficácia do planejamento de seus estudos. Três técnicas podem ser usadas nessa etapa.

Na técnica Estratégias para organização do tempo dos estudos, o acadêmico assinala quais das estratégias apresentadas podem melhor auxiliá-lo na organização do tempo dos estudos, por exemplo: "realizar planejamento diário", "trabalhar com prazos", "usar agenda" etc. Sugere-se que o orientador discuta com o aluno cada uma das estratégias marcadas, relacionando-as 
com a forma como ele planeja implementá-las e discutindo possíveis dificuldades que possam surgir.

A Checklist para organização dos estudos visa ajudar o estudante no planejamento de uma nova agenda, buscando otimizar seus estudos. O material apresenta uma lista com algumas orientações mais diretivas que auxiliam em sua construção, tais como: "identifique o que pode não estar funcionando em sua agenda atualmente", "reserve espaço para descanso e lazer em sua agenda", "realize um planejamento realista". Cada uma dessas orientações pode ser analisada considerando a Agenda real da semana.

A técnica Definindo prioridades: urgente $x$ importante foi adaptada do livro de Wagner (2003) e tem como objetivo contribuir com o estudante na organização das tarefas a serem realizadas. $\mathrm{O}$ aluno deve listar as atividades que precisam ser completadas e classificá-las, dividindo-as conforme os critérios de importância e urgência. Nesse sentido, uma atividade pode ser: urgente e importante; não importante e urgente; não urgente importante; ou não urgente e não importante. No material, são apresentados alguns exemplos de atividades, além da descrição de cada uma destas quatro classificações.

\section{Etapa 3: Estabelecimento de objetivos e metas}

A terceira etapa destina-se a auxiliar o universitário na efetivação das estratégias de mudança, por meio da definição de objetivos de estudo, prioridades (relacionadas a prazos) e metas (curto, médio e longo prazo), considerando a forma como poderá alcançá-las. A partir disso, o estudante poderá planejar sua gestão do tempo de estudos. Nesta etapa, são recomendadas seis diferentes técnicas.

A técnica Definindo objetivos pretende ajudar no estabelecimento de objetivos acadêmicos, pessoais e profissionais, considerando a perspectiva temporal. Pode-se conversar sobre a importância da gestão do tempo para o alcance de objetivos, independentemente de sua natureza. Pretende-se auxiliar o estudante a compreender que os objetivos, apesar de serem divididos por áreas, muitas vezes estão relacionados e sofrem influências mútuas. Assim, ele escreve seus objetivos, classificando-os em curto, médio e longo prazo, considerando seus papéis de vida. O orientador pode auxiliar na reflexão sobre o que é necessário fazer para conseguir alcançá-los ou, se necessário, readequá-los.
A técnica Estabelecendo objetivos SMART de estudos, adaptada do livro de Wagner (2003), apresenta orientações sobre como criar metas claras e precisas, além de algumas dicas que podem ajudar em sua organização. Metas SMART é uma sigla (do inglês) para conseguir definir objetivos de forma mais eficiente. Imaginando que cada letra se refere a uma característica da meta, o S é para específica, M para mensurável, A para atingível, $\mathrm{R}$ para relevante e T para o tempo necessário para sua realização. Esses cinco atributos são considerados essenciais, pois aumentam a probabilidade de alcançar a meta estabelecida.

O Diário de gestão do tempo de estudo pode ser utilizado para que o acadêmico tenha uma visão geral de sua administração do tempo de estudo e de como tem se dedicado para atingir os objetivos propostos. O estudante define metas de estudo a serem cumpridas ao longo da semana. A cada dia, também registra o tempo dedicado para as atividades realizadas durante esse período (estudo, lazer, sono e outras). Solicita-se que ele avalie os resultados alcançados, as metas estabelecidas e as estratégias utilizadas. Para essa etapa, é fundamental que o orientador ressalte que as metas devem ser objetivas e quantificáveis para possibilitar seu monitoramento e realização diária.

A técnica Planejamento de atividades de estudo durante a semana tem como objetivo auxiliar o estudante em sua organização semanal dos estudos. Inicialmente, o acadêmico deverá listar as atividades de estudos, indicando o tempo que estima ser necessário para completá-las. Ao longo da semana, é solicitado que ele registre o tempo realmente utilizado para finalizar as atividades planejadas, os resultados alcançados e a avaliação do que foi feito. Caso o aluno não tenha conseguido realizar alguma das atividades, isto também deverá ser registrado no material. Nessas situações, o orientador poderá refletir com o estudante sobre as dificuldades que podem estar interferindo nas atividades de estudo, além de avaliar a possibilidade de um novo planejamento, se necessário.

$O$ Modelo de planejamento semanal: lista $A B C$ é um material que também foi adaptado do livro de Wagner (2003). Essa técnica se assemelha com a descrita anteriormente, também tendo como objetivo auxiliar no planejamento das atividades semanais de estudo. Além da lista de atividades, o aluno deverá estabelecer a ordem em que devem ser realizadas, indicar o tempo (estimado e real), o status da atividade e a avaliação dos resultados alcançados. A letra 
A se refere àquela tarefa crítica que precisa ser feita imediatamente; a letra B à tarefa que é importante, mas não precisa ser feita imediatamente; a letra $C$ é a tarefa em que não existe urgência no cumprimento, mas que deverá ser feita; e a letra $D$ é a tarefa que pode ser descartada. Tarefas do tipo D serão eliminadas, por isso chamamos o método de Sistema ABC. Esse sistema possibilita que o estudante defina a importância e a urgência de cada tarefa a fim de estabelecer prioridades. O "status da tarefa" permite que o aluno identifique $o$ andamento das atividades por meio de legendas indicadas no material.

O Roteiro de estudo para provaé utilizado para planejar os estudos em uma situação de avaliação específica. O estudante responde questões que irão orientá-lo para o planejamento dos estudos, por exemplo: "quais conteúdos preciso estudar?", "como pretendo estudar?", “quanto tempo acho que será necessário?”. Além disso, registra o que pretende fazer para estudar, para então descrever o que realmente realizou. No final, ele analisa se conseguiu alcançar os objetivos de estudo propostos. É interessante analisar quais foram as facilidades e/ou as dificuldades percebidas ao longo desse processo.

\section{Etapa 4: implementação e avaliação de mudanças}

Por fim, a quarta etapa destina-se à avaliação da gestão do tempo de estudos após o estudante ter definido seus objetivos. Nesse momento, identificam-se quais as principais facilidades e/ou dificuldades para implementação das mudanças propostas. Essa etapa visa ao automonitoramento e à autoavaliação sobre a gestão do tempo de estudos, indicando o que, em sua percepção, já melhorou e o que ainda precisaria mudar. Esse processo de reflexão pode demandar um tempo maior durante $\mathrm{o}$ atendimento, pois alterações e a implementação de outras estratégias de gestão do tempo podem ser necessárias para alcançar os objetivos almejados. Nessa etapa, podem ser utilizadas três técnicas.

A técnica Avaliação dos objetivos SMART tem como propósito que o acadêmico analise os objetivos de estudo estabelecidos, considerando os resultados alcançados (dificuldades e facilidades enfrentadas durante o processo). $\mathrm{O}$ aluno também deve registrar no material quais mudanças que considera necessárias, caso tenha identificado algum empecilho para o alcance do objetivo. Nessas situações, o orientador pode solicitar que o estudante tente identificar qual aspecto do SMART não conseguiu realizar de modo satisfatório (por exemplo: o objetivo não era concreto o suficiente, não foi determinado um tempo específico para se dedicar ao objetivo, o objetivo era de difícil alcance etc.).

O Roteiro de avaliação da gestão do tempo de estudo e a Avaliação do uso do tempo na semana são duas técnicas bastante semelhantes. Apresentam um conjunto de questões com a finalidade de que o universitário reflita sobre sua administração semanal do tempo. A diferença consiste em que o roteiro é destinado ao orientador com perguntas que podem ser feitas durante o atendimento. Já na técnica Avaliação do uso do tempo, o próprio aluno é quem responde as questões por escrito. Alguns exemplos de perguntas são: "como tem considerado seu tempo de estudo?", "quais dificuldades/facilidades foram percebidas ao longo da semana?". Na utilização dessas técnicas, o orientador pode refletir com o aluno sobre seu comprometimento para melhorar sua gestão do tempo de estudos.

\section{Discussões}

As dificuldades em administrar o tempo de estudos de forma satisfatória foram identificadas como uma das principais queixas dos estudantes que buscam o serviço-escola do NAE. Muitos relatam que percebem a necessidade de mudar o modo como estavam acostumados a estudar ao ingressar na universidade. Descrevem que, antes, quando estavam na escola, poderiam apenas estar atentos nas aulas e estudar nas vésperas das provas para obter um bom desempenho. Na universidade, por outro lado, percebem a necessidade de organizar uma rotina de estudos, já que os conteúdos são mais complexos, a carga horária das disciplinas é maior, os prazos são mais rígidos e há a necessidade de gerenciar e conciliar outras atividades, além das acadêmicas (Pinho et al., 2013; Teixeira et al., 2008). O desenvolvimento de competências de gerenciamento do tempo torna-se, portanto, essencial na universidade para o êxito acadêmico e profissional.

O guia abordado neste artigo foi desenvolvido para auxiliar no atendimento de casos que apresentavam essa demanda. Constata-se que o material tem proporcionado uma intervenção organizada e efetiva para gestão do tempo, segundo a percepção dos orientadores. Os profissionais que utilizaram o guia para realizar as intervenções apontaram apenas que gostariam que fosse acrescentado junto ao material uma breve contextualização teórica sobre gestão do tempo na universidade, o que será incluído posteriormente. 
Em relação ao método desenvolvido nesse guia, é possível: 1) diagnosticar as dificuldades que interferem na organização do tempo geral e de estudos, especialmente; 2) propor estratégias de mudança que ampliam o repertório dos alunos atendidos na área de gestão do tempo; 3) proporcionar uma reflexão e o estabelecimento de objetivos acadêmicos e de carreira a curto, médio e longo prazo; e 4) reavaliar as estratégias de gestão do tempo utilizadas para verificar sua eficácia e, talvez, readequá-las conforme a necessidade.

Na primeira etapa do guia, a compreensão de como o indivíduo gerencia seu tempo por meio da visualização das agendas concretas permite o desenvolvimento de compreensões sobre as dificuldades enfrentadas no processo de organização do tempo. A procrastinação foi identificada como um dos principais aspectos relacionados às dificuldades de gerir o tempo dos estudantes que buscaram o serviço do NAE. A procrastinação pode ser definida como o adiamento voluntário e desnecessário de alguma atividade pretendida, mesmo sabendo das potenciais consequências negativas desse atraso (Steel \& Klingsieck, 2016). No âmbito acadêmico, a procrastinação pode resultar na perda dos prazos de execução de tarefas avaliativas (Steel \& Klingsieck, 2016). Entre estudantes universitários, esse comportamento é estimado entre $80 \%$ e $95 \%$, o que evidencia alta incidência desse fenômeno no ensino superior (Steel, 2007).

A aplicação da Agenda real tem possibilitado ao aluno verificar de que forma seu tempo está sendo utilizado de modo geral e na identificação de dificuldades em relação a isso.

Além disso, pode auxiliar até mesmo na compreensão dos papéis de vida que o aluno desempenha nesse período de ingresso no ensino superior. $\mathrm{O}$ estilo de vida do indivíduo é influenciado pela conjunção de diferentes papéis que ele ocupa (por exemplo: filho(a), estudante, trabalhador(a), pai/mãe etc.). Assim, é importante lembrar que o indivíduo não se restringe unicamente a seu papel de estudante universitário; ele desenvolve diferentes papéis de vida que devem ser considerados na organização dos seus estudos. Desse modo, a gestão do tempo na universidade é fundamental não somente para o desempenho e sucesso acadêmico, mas para que o indivíduo possa experimentar bem-estar em sua vida de maneira geral (Blando, 2015).

Em vários atendimentos, também se observou que o preenchimento da Agenda real permitiu que os alunos constatassem que estavam dedicando menos tempo do que gostariam aos estudos e que o tempo de lazer e uso de tecnologias, normalmente, sobressaiam. Em um estudo realizado com uma amostra de universitários brasileiros, verificou-se que o uso de tecnologias (principalmente internet e celular) ultrapassava o valor de 60 horas semanais, sendo que muitos acabavam se privando de necessidades fisiológicas (dormir, alimentar-se, ir ao banheiro) ou se descuidavam de tarefas acadêmicas, domésticas ou trabalho para poder permanecer mais tempo usando a tecnologia (Silva \& Vizzotto, 2013). Em outros casos atendidos no serviço, verificou-se o inverso: os estudos predominavam e havia pouco tempo reservado ao lazer e projetos pessoais. Em ambas as situações, os estudantes que não conseguem distribuir adequadamente as atividades no tempo podem enfrentar dificuldades durante o ensino superior, além de estarem mais propensos ao desenvolvimento de sintomatologias relacionadas a estresse, ansiedade e depressão (Oliveira et al., 2016). Em contrapartida, boas práticas de manejo do tempo são relacionadas a resultados positivos na vida acadêmica, uma vez que a percepção de maior controle ajuda os estudantes a ficarem menos estressados e a se tornarem mais eficientes e satisfeitos, tanto com sua vida quanto com seu processo de aprendizagem (Häfner, Stock, Pinneker, \& Ströhle, 2014; Leite, Tamayo, \& Günther, 2003).

Observou-se que os alunos atendidos no serviço que colocavam as atividades acadêmicas como as únicas tarefas prioritárias, em geral, sentiam que estavam deixando de lado outros aspectos importantes de suas vidas (tais como família, lazer, sono, alimentação e cuidados pessoais). Já aqueles que deixavam os estudos para o último momento relatavam insatisfação com seu processo de aprendizagem, tendo resultados acadêmicos inferiores aos desejados. Nesse sentido, é importante que o estudante reflita sobre seu envolvimento nas atividades acadêmicas de modo a redimensionar o tempo dedicado aos diversos aspectos de sua vida, favorecendo, assim, o engajamento e execução das diferentes tarefas (Basso et al., 2013).

A segunda etapa do guia tem possibilitado que o aluno proponha estratégias para organização dos estudos, considerando os problemas de gerenciamento das atividades no tempo averiguadas previamente. As técnicas presentes nessa etapa proporcionam um repertório de estratégias e visam ajudar o estudante no planejamento de uma nova agenda, buscando otimizar seus estudos. O papel do orientador nessa etapa é o de auxiliar na reflexão da relação entre as dificuldades da 
gestão do tempo e a forma de saná-las. Somente a partir disso será possível desenvolver e estabelecer estratégias mais efetivas e que repercutam nos processos de aprendizagem. Além disso, por meio da técnica Definindo prioridades: urgente $x$ importante, o estudante consegue definir prioridades, diferenciando e relacionando a urgência com a importância que essas tarefas tem em sua vida. Ao longo dos atendimentos, percebe-se que os estudantes costumam ter dificuldades em estabelecer prioridades, tendo em vista que, ao deixar tarefas acadêmicas para a última hora, entendem que tudo é urgente e importante. Além disso, se em seu cotidiano o estudante não souber e não conseguir definir prioridades, poderá acabar por iniciar todas as atividades e não concluir nenhuma, prejudicando seu senso de produtividade e, consequentemente, $\mathrm{o}$ alcance de seus objetivos (Wagner, 2003).

No início dos atendimentos, identificou-se também que muitos alunos realizavam um planejamento dos estudos, mas que não eram compatíveis com sua disponibilidade e nem consideravam o tempo despendido em outros papéis de vida. Por exemplo, alguns preenchiam todo o tempo livre com estudos e, consequentemente, acabavam se sentido frustrados por não conseguirem cumprir o cronograma conforme o planejado. Para propor uma organização das atividades de estudos, é preciso, primeiramente, avaliar o modo como o tempo é realmente utilizado. Somente após esta análise é possível propor e implementar estratégias condizentes com a realidade (Blando, 2015; Durán-Aponte \& Pujol, 2013; GarcíaRos \& Pérez-Gonzáles, 2012).

A terceira etapa do guia enfatiza a importância de trabalhar a questão do comprometimento, ou seja, o que se pretende e o que realmente se vai fazer para atingir os objetivos de estudo. Ressalta-se, novamente, que, se os objetivos que o estudante almeja alcançar não estiverem claros e definidos, o comprometimento poderá ser prejudicado. Para que objetivos possam ser bem definidos, eles devem ser o mais específicos e realistas possível (por exemplo, em vez de "estudar mais tempo para a disciplina x", transformá-lo em "fazer quinze exercícios da disciplina $\mathrm{x}$, por duas horas por dia ao longo da semana"). Dessa forma, aumenta-se a chance de que estes objetivos sejam alcançados e que se obtenham resultados. Isso porque a tarefa tende a se tornar mais palpável e possível de realizar. Além disso, é importante que o aluno estabeleça critérios para avaliar o alcance de suas metas e objetivos, e que, ao planejar suas atividades de estudos, considere o próprio ritmo, a quantidade de disciplinas, os horários mais apropriados para a realização dos estudos, a necessidade de períodos de lazer e de descanso, e outros relacionados aos demais papéis de vida (Blando, 2015; Oliveira et al., 2016).

Na universidade, o gerenciamento do tempo de modo adequado é essencial para o sucesso acadêmico e para o alcance dos objetivos estabelecidos (GarcíaRos \& Pérez-Gonzáles, 2012; Gárcia-Ros et al., 2008). Estudos indicam que um bom uso do tempo por parte dos estudantes apresenta impacto positivo no desempenho acadêmico (Umerenkova \& Flores, 2018). Seu propósito, contudo, não se restringe ao aumento da produtividade na execução das tarefas acadêmicas, mas considera também a qualidade do tempo envolvido nas diferentes atividades, de modo a proporcionar bem-estar, redução do estresse e maior satisfação com a aprendizagem (Leite et al., 2003, Pellegrini, Calais, \& Salgado, 2012).

Finalmente, a quarta etapa apresentada no guia tem proporcionado a reflexão, tanto do orientador quanto do aluno, sobre a efetividade das estratégias de mudanças praticadas para otimizar o tempo de estudos. É essencial que o estudante continue observando o uso do tempo nas diferentes atividades. Monitorar e analisar os resultados alcançados permite identificar a satisfação com o desempenho nas atividades. Além disso, possibilita verificar se ainda existem obstáculos para o alcance dos objetivos almejados a fim de propor novas mudanças, se necessário (García-Ros \& Pérez-Gonzáles, 2012). Esse monitoramento pode ser traduzido como uma auto-observação das ações em sua gestão do tempo e no modo como isso pode afetar seus estudos e desempenho (Panadero \& Alonso-Tapia, 2014). Entretanto ressalta-se que, apesar da análise da quantidade de horas despendidas ser um fator importante para a organização do processo de aprendizagem, ela não explica, isoladamente, o bom desempenho acadêmico, já que é preciso avaliar também a dimensão qualitativa do estudo realizado (Veiga \& Melim, 2007; Umerenkova \& Flores, 2018). Ademais, deve-se enfatizar o quanto não existe uma "receita mágica" para a resolução de problemas associados ao tempo. Ou seja, o estudante precisará identificar suas próprias dificuldades e recursos (facilidades na administração do tempo) para estabelecer e experimentar estratégias que melhor se adequem a seu funcionamento e a seu contexto de vida (Basso et al., 2013; Oliveira et al., 2016). 
É fundamental ressaltar que se espera que o atendimento de orientação à aprendizagem para a gestão do tempo de estudos possibilite que o acadêmico consiga desenvolver sua capacidade de autorreflexão sobre o processo, agindo de forma ativa e autônoma em sua aprendizagem na universidade (Blando, 2015). Desse modo, a prática da gestão do tempo, mesmo após a finalização do atendimento, deverá seguir como um processo contínuo em que o universitário deverá se automonitorar, autoavaliar e implementar mudanças em sua organização de estudos quando for necessário.

Ao final do atendimento de orientação à aprendizagem em relação à gestão do tempo de estudos, é solicitado que o aluno faça uma avaliação verbal de todo o processo. Em geral, os estudantes relataram se sentir mais comprometidos e dedicados aos estudos e consideraram úteis as técnicas que se propõem a planejar e a estabelecer objetivos semanais a serem atingidos. Um dos estudantes atendidos considerou que obteve mudanças em seu processo de estudos graças à gestão do tempo:

Primeiro para me organizar melhor, ver que tenho horários disponíveis. Organizei melhor o ambiente de estudo. Também, o que acho que mais ajudou, dividi em metas uma tarefa que era muito grande e sempre que pensava já me cansava, não tinha nem coragem de começar (Estudante 1).

Além disso, outros estudantes comentaram que conseguem perceber mudanças no próprio comportamento e que ter os estudos como rotina tornou-se um hábito mais frequente ao longo das semanas. Também revelaram que passaram a procrastinar menos a partir da implementação das estratégias pensadas no atendimento. Por fim, uma estudante revelou que o trabalho de gestão do tempo de estudos o auxiliou para além de questões acadêmicas: "o trabalho foi muito bom! Ajuda a pensar o que está fazendo, diariamente não só o estudo, mas também no trabalho e lazer. Abre um leque de possibilidades para alcançar teu objetivo na vida" (Estudante 2). Não foram apresentados, por parte dos estudantes que participaram do atendimento voltado à gestão do tempo, pontos negativos em relação às intervenções, exceto alguns que consideraram necessário melhorar a divulgação realizada sobre o trabalho oferecido.

\section{Considerações finais}

Este estudo teve como objetivo apresentar a construção do Guia de técnicas de gestão do tempo de estudo na universidade, desenvolvido a partir da prática de atendimentos em orientação à aprendizagem no NAE da UFRGS. Foi possível observar que sua utilização no serviço tem conseguido auxiliar os orientadores no atendimento a estudantes com dificuldades na gestão do tempo de estudos. Ademais, o guia tem auxiliado os orientadores em formação, possibilitando maior repertório de técnicas a serem utilizadas no atendimento, além de melhor entendimento do processo que envolve a relação da organização de estudos com a gestão do tempo.

Ao compreender a gestão do tempo de estudos como processo, o guia foi desenvolvido com a finalidade de criar um método de atendimento a partir das etapas apresentadas, o que inclui entender os problemas de organização do tempo trazido pelo estudante, propor estratégias para resolvê-los, implementar e avaliar mudanças e, por fim, realizar alterações quando necessário. O material não pretende ser prescritivo, pois é necessário compreender a situação de cada estudante em relação às dificuldades de gestão do tempo, de modo a adaptar as técnicas a partir de sua realidade. Ademais, compreende-se que auxiliar os alunos em sua gestão do tempo de estudos não é um processo simples, pois envolve considerar outros aspectos da vida além da dimensão acadêmica, tais como questões pessoais e profissionais. Nesse sentido, o objetivo do atendimento é auxiliar o universitário a refletir sobre sua administração do tempo, contribuindo para o desenvolvimento da autonomia em relação a sua aprendizagem. Ressalta-se, no entanto, que, apesar de o trabalho desenvolvido ser focado no indivíduo, considera-se que cabe também às instituições de ensino superior repensar as formas como são estruturados seus cursos e currículos, bem como seu ensino e métodos de avaliação com vistas a não contribuir com as dificuldades de gestão do tempo que o estudante pode enfrentar ao longo de sua trajetória acadêmica.

Apesar de sua proposta inovadora, é importante frisar que o guia está sendo utilizado recentemente no serviço e ainda é incipiente a avaliação da efetividade de cada técnica. Futuramente, pretende-se desenvolver formas de avaliação sistemática, de modo a analisar sua contribuição tanto para os orientadores como os universitários. Por fim, entende-se que o guia 
abordado neste trabalho pode servir como modelo para o desenvolvimento de novos materiais destinados a profissionais e pesquisadores que trabalham com universitários. Informações sobre como ter acesso ao guia podem ser requisitadas para as duas primeiras autoras deste artigo.

\section{Referências}

Basso, C., Graf, L. P., Lima, F. C., Schmidt, B., \& Bardagi, M. P. (2013). Organização do tempo e métodos de estudos: Oficinas com estudantes universitários. Revista Brasileira de Orientação Profissional, 14(2), 277-288.

Blando, A. (2015). Dificuldades acadêmicas que interferem na aprendizagem de estudantes universitários de engenharia e de ciências exatas: Um estudo fundamentado na epistemologia genética [Dissertação de mestrado, Universidade Federal do Rio Grande do Sul]. Repositório digital da UFRGS. https://lume.ufrgs.br/handle/10183/115722

Briggs, A. R., Clark, J., \& Hall, I. (2012). Building bridges: Understanding student transition to university. Quality in Higher Education, 18(1), 3-21. http://dx.doi.org/10.1080/13538322.2011.614468

Claessens, B. J. C., Van Eerde, W., Rutte, C. G., \& Roe, R. A. (2007). A review of the time management literature. Personnel Review, 36(2), 255-276. http://dx.doi.org/10.1108/00483480710726136

Ding, F. (2017). "Free in time, not free in mind": First-year university students becoming more independent. Journal of College Student Development, 58(4), 601-617. http://dx.doi.org/10.1353/csd.2017.0045

Durán-Aponte, E., \& Pujol, L. (2013). Manejo del tiempo académico en jóvenes que inician estudios en la Universidad Simón Bolívar. Revista Latinoamericana de Ciencias Sociales, Niñez y Juventud, 11(1), 93-108. http://www.redalyc.org/articulo.oa?id=77325885022

García-Ros, R., \& Pérez-González, F. (2012). Spanish version of the Time Management Behavior Questionnaire for university students. The Spanish Journal of Psychology, 15(3), 1485-1994. http://dx.doi.org/10.5209/ rev_SJOP.2012.v15.n3.39432

García-Ros, R., Pérez-González, F., Talaya, I., \& Martínez, E. (2008). Análisis de la gestión del tiempo académico de los estudiantes de nuevo ingreso en la titulación de Psicología: Capacidad predictiva y análisis comparativo entre dos instrumentos de evaluación. International Journal of Developmental and Educational Psychology, 2(1), 245-252.

Häfner, A., Stock, A., Pinneker, L., \& Ströhle, S. (2014). Stress prevention through a time management training intervention: An experimental study. Educational Psychology, 34, 403-416. http://dx.doi.org/10.1080/01443410.2013.785065

Leite, U. R., Tamayo, A., \& Günther, H. (2003). Organização do uso do tempo e valores de universitários. Avaliação Psicológica, 2(1), 57-66.

Oliveira, C. T., Carlotto, R. C., Teixeira, M. A. P., \& Dias, A. C. G. (2016). Oficinas de Gestão do Tempo com Estudantes Universitários. Psicologia: Ciência e Profissão, 36(1), 224-233. http://dx.doi.org/10.1590/1982-3703001482014

Panadero, E., \& Alonso-Tapia. (2014). Cómo autorregulan nuestros alumnos? Revisión del modelo cíclico de Zimmerman sobre autorregulación del aprendizaje. Anales de Psicología, 30(4), 450-462.

Pavão, S. M. O., \& Cezar, A. P. F. (2015). Educação superior: Vicissitudes da ação psicopedagógica. Revista Psicopedagogia, 32(99), 366-373.

Pellegrini, C. F. S., Calais, S. L., \& Salgado, M. H. (2012). Habilidades sociais e administração de tempo no manejo do estresse. Arquivos Brasileiros de Psicologia, 64(3), 110-129.

Pinho, A. P. M., Bastos, A. V. B., Dourado, L. C., \& Ribeiro, J. L. L. S. (2013). A transição do ensino médio para a universidade: Um estudo qualitativo sobre os fatores que influenciam este processo e suas possíveis consequências comportamentais [Artigo apresentado]. Anais do XIII Coloquio de Gestión Universitária en Américas, Florianópolis, SC, Brasil. https://repositorio.ufsc.br/handle/123456789/113099

Silva, F. C., \&Vizzotto, M. M. (2013). Perfil do estudante universitário usuário de tecnologias. Psicólogo InFormação, 17(17), 39-55. http://dx.doi.org/10.15603/2176-0969/pi.v17n17p39-55

Steel, P. (2007). The nature of procrastination:A meta-analytic and theoretical review of quintessential self-regulatory failure. Psychological Bulletin, 133(1), 65-94. http://dx.doi.org/10.1037/0033-2909.133.1.65

Steel, P., \& Klingsieck, K. B. (2016). Academic procrastination: Psychological antecedents revisited. Australian Psychologist, 51(1), 36-46. http://dx.doi.org/10.1111/ap.12173

Teixeira, M. A. P., Dias, A. C. G., Wottrich, S. H., \& Oliveira, A. M. (2008). Adaptação à universidade em jovens calouros. Psicologia Escolar e Educacional, 12(1), 185-202. 
Thibodeaux, J., Deutsch, A., Kitsantas, A., \&Winsler, A. (2017). First-year college students time use: Relations with selfregulation and GPA. Journal of Advanced Academics, 28(1), 5-27. http://dx.doi.org/10.1177/1932202X16676860

Umerenkova, A. G., \& Flores, J. G. (2018). Gestión del tiempo en alumnado universitario con diferentes niveles de rendimiento académico. Educação e Pesquisa, 44, e157900. http://dx.doi.org/10.1590/s1678-4634201708157900

Veiga, F. H., \& Melim, A. C. (2007). Questionário de gestão do tempo académico em alunos do ensino básico e secundário: Adaptação Portuguesa do Time Management Questionnaire. Psicologia Educação Cultura, 11(2), 329-341.

Wagner, J. (2003). A arte de planejar o tempo. Literalis.

White, S. M., Riley, A., \& Flom, P. (2013). Assessment ofTime Management Skills (ATMS): A practice-based outcome questionnaire. Occupational Therapy in Mental Health, 29(3), 215-231. http://dx.doi.org/10.1080/0164212X.2013.819481

Zoltowski, A. P. C. (2016). Autorregulação da aprendizagem: Levantamento e intervenção com estudantes universitários [Tese de doutorado]. Universidade Federal do Rio Grande do Sul.

\section{Fabiane Cristina Pereira Marcilio}

Mestre em Psicologia e Saúde pela Universidade Federal de Ciências da Saúde de Porto Alegre (UFCSPA). Psicóloga e colaboradora no Núcleo de Apoio ao Estudante da Universidade Federal do Rio Grande do Sul (UFRGS), Porto Alegre - RS. Brasil.

E-mail: fabiane_marcilio@hotmail.com

(1) https://orcid.org/0000-0003-2695-6628

\section{Alessandra Blando}

Doutoranda em Educação pela UFRGS. Pedagoga do Núcleo de Apoio ao Estudante da mesma instituição, Porto Alegre - RS. Brasil.

E-mail: alessandra.blando@ufrgs.br

(1) https://orcid.org/0000-0001-5256-5984

\section{Roberta Zanini da Rocha}

Doutoranda em Psicologia pela UFRGS. Psicóloga, Porto Alegre - RS. Brasil.

E-mail: robertazrocha@gmail.com

(1) https://orcid.org/0000-0003-2646-3671

\section{Ana Cristina Garcia Dias}

Doutora em Psicologia pela Universidade de São Paulo (USP). Professora no Programa de Pós-Graduação em Psicologia da UFRGS. Bolsista de produtividade CNPq 2. Psicóloga, Porto Alegre - RS. Brasil.

E-mail: anacristinagarciadias@gmail.com

(1) https://orcid.org/0000-0003-2312-3911

Endereço para envio de correspondência:

Universidade Federal do Rio Grande do Sul. Rua Ramiro Barcelos, 2.777 - Anexo I Saúde, Sala 314. CEP: 90035-003.

Porto Alegre - RS. Brasil.

Recebido 07/01/2019

Aceito 25/03/2020

Received 01/07/2019

Approved 03/25/2020

Recibido 07/01/2019

Aceptado 25/03/2020 
Marcilio, F. C. P., Blando, A., Rocha, R. Z., \& Dias, A. C. G. (2021). Guia de técnicas para a gestão do tempo de estudos.

Como citar: Marcilio, F. C. P., Blando, A., Rocha, R. Z., Dias, A. C. G. (2021). Guia de técnicas para a gestão do tempo de estudos: Relato da construção. Psicologia: Ciência e Profissão, 41, 1-13. https://doi.org/10.1590/ 1982-3703003218325

How to cite: Marcilio, F. C. P., Blando, A., Rocha, R. Z., Dias, A. C. G. (2021). Guide of techniques for study time management: Report of construction. Psicologia: Ciência e Profissão, 41, 1-13. https://doi.org/10.1590/ $1982-3703003218325$

Cómo citar: Marcilio, F. C. P., Blando, A., Rocha, R. Z., Dias, A. C. G. (2021). Guía de técnicas para la gestión del tiempo de estudios: Relato de la construcción. Psicologia: Ciência e Profissão, 41, 1-13. https://doi.org/10.1590/ 1982-3703003218325 